\title{
Development of SNP-based dCAPS markers for identifying male sterile gene $t m s 5$ in two-line hybrid rice
}

\author{
F.S. Song ${ }^{1}$, J.L. Ni ${ }^{1}$, Y.L. Qian ${ }^{2}$, L. Li $^{1}$, D.H. Ni ${ }^{1}$ and J.B. Yang ${ }^{1}$ \\ ${ }^{1}$ Key Laboratory of Rice Genetics Breeding of Anhui Province, \\ Rice Research Institute, Anhui Academy of Agricultural Sciences, Hefei, China \\ ${ }^{2}$ Tobacco Research Institute, Anhui Academy of Agricultural Sciences, \\ Hefei, China \\ Corresponding authors: D.H. Ni / J.B. Yang \\ E-mail: dahuni1974@163.com / yjianbo3@163.com
}

Genet. Mol. Res. 15 (3): gmr. 15038512

Received February 1, 2016

Accepted April 11, 2016

Published August 29, 2016

DOI http://dx.doi.org/10.4238/gmr.15038512

Copyright (C) 2016 The Authors. This is an open-access article distributed under the terms of the Creative Commons Attribution ShareAlike (CC BY-SA) 4.0 License.

\begin{abstract}
Molecular markers can increase both the efficiency and speed of breeding programs. Functional markers that detect the functional mutations causing phenotypic changes offer a precise method for genetic identification. In this study, we used newly derived cleaved amplified polymorphic sequence markers to detect the functional mutations of tms 5 , which is a male sterile gene that is widely used in rice production in China. In addition, restriction cutting sites were designed to specifically digest amplicons of tms 5 but not wild type (TMS5), in order to avoid the risk of false positive results. By optimizing the condition of the polymerase chain reaction amplifications and restriction enzyme digestions, the newly designed markers could accurately distinguish between tms 5 and TMS5. These markers can be applied in marker-assisted selection for breeding novel
\end{abstract}


thermo-sensitive genic male sterile (TGMS) lines, as well as to rapidly identify the TGMS hybrid seed purity.

Key words: Marker-assisted selection; tms 5 gene; dCAPS; Two-line rice; Purity detection

\section{INTRODUCTION}

Discovery and successful application of two-line male sterile rice germplasm resources play important roles in ensuring food safety in China (Dong et al., 2000; Fan and Zhang, 2014). Compared with three-line hybrid rice, two-line hybrid rice is not limited by restore gene(s) and maintainer gene(s), which widely broadens the range of germplasm resources (Nas et al., 2005; Peng et al., 2010; Qi et al., 2014). Therefore, two-line hybrid rice, in general, is superior to threeline hybrid rice, both in quality and in yield. Moreover, the self-reproduction characteristic of two-line male sterile lines simplifies the breeding procedure. Consequently, the growing area of two-line hybrid rice has expanded rapidly in China in recent years (Xu et al., 2011).

Based on the sources of male sterile genes, the two-line rice varieties widely used in production can be divided into two types; photo-sensitive genic male sterile (PGMS) derived from Nongken 58S (Shi, 1985), and thermo-sensitive genic male sterile (TGMS) derived from Annong S-1 (Deng et al., 1999). A single copy gene for PGMS has been cloned by Ding et al. (2012) and Zhou et al. (2012), in which a point mutation of a cytidine-5'-triphosphate in the wild type gene $P M S 3$ was replaced by a guanosine-5'-triphosphate in the mutated gene pms3 (or p/tms12-1), which resulted in the sterility of Nongken 58S. As reported by Zhou et al. (2014), in TGMS, a cytidine-5'-triphosphate in the wild type gene (TMS5) was substituted with an adenosine-5'triphosphate in the mutated tms 5 within a $71 \mathrm{bp}$ coding region. This led to the sterility of Annong S-1 under high temperature. TMS5 encodes RNase Z, while tms 5 is a loss-of-function mutant.

Obviously, pms 3 and tms 5 are key genes for breeding of two-line hybrid rice. Use of molecular markers on these two genes can improve both the efficiency and speed of the selection/breeding programs. Based on the functional mutation of pms 3 , a cleaved amplified polymorphic sequence (CAPS) marker was developed to detect the PGMS allele (Ding et al., 2012). For detection of the TGMS allele, Yang et al. (2011) developed an insertion/deletion (InDel) marker named SJ001. However, the InDel marker is located about $5 \mathrm{kbp}$ away from the mutated site of tms 5. Some inbred rice lines, such as Xieqingzao B, showed false positive results by this marker. Another InDel marker, RMZ-13, designed by Cao et al. (2011), is based on a six-base insertion that is located upstream of the start codon ATG in the tms 5 gene. This insertion was not the functional mutation of TMS5. According to sequencing results reported by Zhang et al. (2014a), many varieties of inbred rice, including 08EZ01, Hua201, Hua966, L718, R287, R288, Yi R88, and Zaoxian2430, showed false positive results after detection by the InDel marker RMZ-13. The authors also found three haplotypes, GC, TC, and TA at 70-71 $\mathrm{bp}$ of the RNase $Z$ coding region. Among these rice lines studied by Zhang et al. (2014a), GC and TC were fertile, whereas TA serves as a tms 5 mutant that causes rice sterility. Zhang et al. (2014a) then developed two derived CAPS (dCAPS) markers (RZ2F1/R and RZ2F2/R), in which restriction enzymes HinfI and StyI were chosen to digest amplicons of wild type lines TC and GC, respectively, but not the TA mutation. In other words, if there were other haplotypes at this locus, HinfI and StyI would be unable to digest them, which still poses a potential risk of false positive detection. However, it is inconvenient to amplify and digest

Genetics and Molecular Research 15 (3): gmr.15038512 
twice for each sample to infer its genotype, and the scoring of the dCAPS markers is often complicated due to incomplete digestion of amplicons (Zhang et al., 2014b).

In this study, we developed dCAPS markers in which a restriction endonuclease specifically digests amplicons of tms5, rather than the wild type, to avoid any false positive detection. The new dCAPS markers can rapidly and accurately distinguish the tms 5 and TMS5 alleles through a single one-time amplification and digestion. These dCAPS markers can be applied in marker-assisted selection and purity identification for hybrid rice seeds.

\section{MATERIAL AND METHODS}

\section{Plant materials and growth conditions}

In this study, we used 213 germplasm lines that included 15 TGMS lines and 198 non-TGMS lines. In addition, we used an $\mathrm{F}_{2}$ population and two hybrid varieties. The 15 TGMS lines included 1892s, MengS, Xin 2S, Guangzhan 63S, Xuan 69S, Guangmo S, Y58S, P88S, Feng 39S, Annong S-1, N422s, H9802s, Xinan S, Shen 08s, and C815S. All these lines were sequenced for the $R N Z$ gene (LOC Os02g12290) and were shown to be male sterile when flowering in summer (July-August) in Hefei city, China; or partially male fertile in spring (mid-February-March) in the Hainan Province, China. Of the 198 non-TGMS lines, restorer lines Yangdao 6 and RH003 originated from Rice Research Institute, Anhui Academy of Agricultural Sciences, whereas the remaining lines came from the USDA Rice Mini-Core Collection (Agrama et al., 2009; Li et al., 2010).

The seeds of hybrid rice varieties Huiliangyou 6 (1892s x Yangdao 6) and Wandao 153 (1892s x RH003) were supplied by Huaan Seed Co., Ltd., China. Two hundred plants per variety were used for purity identification. An $\mathrm{F}_{2}$ population from the cross of $1892 \mathrm{~s}$ and Yangdao 6 were tested for co-segregation of the TGMS trait using designed dCAPS markers. All rice lines were planted in the paddy fields of Anhui Academy of Agricultural Sciences in Hefei city, China. The leaves of seedlings were collected for DNA extraction following the method of Li et al. (2013). The flowering stage appeared in August (average temperature $>25^{\circ} \mathrm{C}$ ) and panicles of each plant were collected for microscopic examination after staining with $\mathrm{I}_{2}$-KI solution following Zhou et al. (2014).

\section{Primer design and polymerase chain reaction (PCR) amplification}

Primers were designed using the dCAPS Finder 2.0 software (Neff et al., 2002). Since no restriction site adjacent to the mutation site was available, mismatches were introduced into the primers. This was done to create restriction recognition sites on the amplicons of the tms 5 allele only, but not that of the TMS5 allele.

Moreover, based on the availability and the cost of restriction enzymes, seven selective primers with cutting sites corresponding to endonucleases MaeI, MboII, XbaI, RsaI, AluI, ScaI, and SnaI were chosen (S1-S7 in Table 1). Among these, S1, S2, and S3 align to the sense strand of the gene, whereas S4, S5, S6, and S7 align to the anti-sense strand. These restriction enzymes targeted four primers (T1-T4; Table 1), resulting in 14 combinations of PCR amplifications (Table 2). Three additional target primers (T5, T6, and T7; Table 1) were designed by Primer Premier 5 software (http://www.premierbiosoft.com). All primers were commercially synthesized by Invitrogen Biotechnology Co., Ltd., Shanghai, China.

Genetics and Molecular Research 15 (3): gmr.15038512 
Table 1. Sequence, mismatch numbers, and mismatch positions of the designed dCAPS primers.

\begin{tabular}{|c|c|c|c|c|c|}
\hline Type & Name & Primer sequence $\left(5^{\prime} \text { to } 3^{\prime}\right)^{\mathrm{a}}$ & Mismatch No. & Mismatch position $^{\mathrm{b}}$ & Direction $^{\mathrm{c}}$ \\
\hline \multirow[t]{7}{*}{ Selective primers } & S1 & CCGCGCCGCCACCGGGTCGGCCGAAC T & 1 & 2 & Sense \\
\hline & S2 & CCGCGCCGCCACCGGGTCGGCCGAAGA & 1 & 1 & Sense \\
\hline & S3 & CCGCGCCGCCACCGGGTCGGCCGATCT & 2 & 2 and 3 & Sense \\
\hline & S4 & CTCGACGGTGAGGGGCGGCGCCTT $\underline{G}$ & 1 & 1 & Anti-sense \\
\hline & S5 & CTCGACGGTGAGGGGCGGCGCCAGC & 2 & 2 and 3 & Anti-sense \\
\hline & S6 & CTCGACGGTGAGGGGCGGCGCCTAG & 2 & 2 and 3 & Anti-sense \\
\hline & S7 & CTCGACGGTGAGGGGCGGCGCC $\underline{\underline{T}} \underline{\underline{A}}$ & 2 & 1 and 2 & Anti-sense \\
\hline \multirow[t]{7}{*}{ Target primers } & $\mathrm{T} 1$ & CGGGAAGATGACGCAGGT & No & No & Anti-sense \\
\hline & $\mathrm{T} 2$ & CGCTCAGCGTCGGGAAGA & No & No & Anti-sense \\
\hline & T3 & GGCGAACAGCGGCAAGTC & No & No & Sense \\
\hline & T4 & GAACAGCGGCAAGTCATCG & No & No & Sense \\
\hline & $\mathrm{T} 5$ & GCGGCGGCGCCATGGCGAACA & No & No & Sense \\
\hline & T6 & GTGCCGGAACAACCCCCCACCACGG & No & No & Sense \\
\hline & $\mathrm{T} 7$ & CCGGCCCATCGTGCTTCGTGCCAAA & No & No & Sense \\
\hline
\end{tabular}

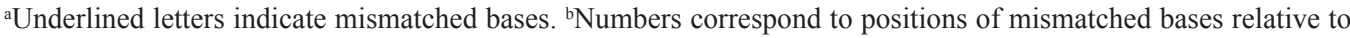
the 3' end of the selective primers. "Sense" means that primer sequence aligns to the sense strand of the gene, while "anti-sense" means that primer sequence aligns to the anti-sense strand of the gene.

Table 2. Primer sets, expected product size, restriction enzymes, and recognition sites of the designed dCAPS primers.

\begin{tabular}{|c|c|c|c|c|}
\hline Primer set No. & Primer pair (selective + target) & Expected product (bp) & Recognition site & Restriction enzyme \\
\hline 1 & $\mathrm{~S} 1+\mathrm{T} 1$ & $110 / 83$ & CTAG & MaeI \\
\hline 2 & $\mathrm{~S} 1+\mathrm{T} 2$ & $120 / 93$ & CTAG & MaeI \\
\hline 3 & $\mathrm{~S} 2+\mathrm{T} 1$ & $110 / 72$ & GAAGA & MboII \\
\hline 4 & $\mathrm{~S} 2+\mathrm{T} 2$ & $120 / 82$ & GAAGA & MboII \\
\hline 5 & $\mathrm{~S} 3+\mathrm{T} 1$ & $110 / 85$ & TCTAGA & $X b a \mathrm{I}$ \\
\hline 6 & $\mathrm{~S} 3+\mathrm{T} 2$ & $120 / 95$ & TCTAGA & $X b a \mathrm{I}$ \\
\hline 7 & $\mathrm{~S} 4+\mathrm{T} 3$ & $94 / 68$ & GTAC & $R s a \mathrm{I}$ \\
\hline 8 & $\mathrm{~S} 4+\mathrm{T} 4$ & $91 / 65$ & GTAC & $R s a \mathrm{I}$ \\
\hline 9 & $\mathrm{~S} 5+\mathrm{T} 3$ & $94 / 70$ & AGCT & AluI \\
\hline 10 & $\mathrm{~S} 5+\mathrm{T} 4$ & $91 / 67$ & AGCT & AluI \\
\hline 11 & $\mathrm{~S} 6+\mathrm{T} 3$ & $94 / 68$ & AGTACT & ScaI \\
\hline 12 & S6+T4 & $91 / 65$ & AGTACT & ScaI \\
\hline 13 & $\mathrm{~S} 7+\mathrm{T} 3$ & $94 / 69$ & GTATAC & SnaI \\
\hline 14 & $\mathrm{~S} 7+\mathrm{T} 4$ & $91 / 66$ & GTATAC & SnaI \\
\hline
\end{tabular}

\section{PCR amplifications and restriction enzyme digestions}

Our results suggested several CpG islands in TMS5 (see below). CpG islands often limit PCR amplifications. A CpG island is defined by $>65 \% \mathrm{G}+\mathrm{C}$ content with a GC-rich region $>200 \mathrm{bp}$. In order to obtain ideal amplification results of tms 5 , PCR conditions were optimized. Two DNA polymerases ( $r$ Taq and PrimeSTAR HS DNA polymerase) and five PCR buffers (10X PCR buffer, 2X GC buffer I, 2X GC buffer II, 5X PrimeSTAR buffer, and 2X PrimeSTAR GC buffer, all buffers contained $\mathrm{Mg}^{2+}$ ) were purchased from Takara Biotechnology Co., Ltd., Dalian, China. The settings for the tms 5 PCR screenings followed the manufacturer recommendations.

For PCR condition 1 (regular PCR amplification), the amplification was conducted in a $25.0 \mu \mathrm{L}$ volume, containing $2.5 \mu \mathrm{L} 10 \mathrm{X}$ PCR buffer, $1.6 \mu \mathrm{L}$ dNTPs $(2.5 \mathrm{mM}$ each $), 1.0 \mu \mathrm{L}$ each primer $(10 \mu \mathrm{M}), 0.2 \mu \mathrm{L} r \operatorname{Taq}(5 \mathrm{U} / \mu \mathrm{L}), 1.0 \mu \mathrm{L}$ genomic DNA $(50 \mathrm{ng} / \mu \mathrm{L})$, and $\mathrm{dH}_{2} \mathrm{O}$ to reach the target volume of $25 \mu \mathrm{L}$. For conditions 2 and 3, the 10X PCR buffer was replaced with $12.5 \mu \mathrm{L} 2 \mathrm{X}$ GC buffer I, or $12.5 \mu \mathrm{L} 2 \mathrm{X}$ GC buffer II, respectively. All other reagents were the same as in condition 1, with the exception of the $\mathrm{dH}_{2} \mathrm{O}$ volume adjustment. In condition 
4, $5.0 \mu \mathrm{L} 5 \mathrm{X}$ PrimeSTAR buffer and $0.25 \mu \mathrm{L}$ PrimeSTAR HS DNA polymerase $(2.5 \mathrm{U} / \mu \mathrm{L})$ were used to replace the 10X PCR buffer and $0.2 \mu \mathrm{L} \mathrm{rTaq}$ in condition 1, while all other components were the same as in condition 1 except the $\mathrm{dH}_{2} \mathrm{O}$ volume. In condition $5,12.5 \mu \mathrm{L}$ $2 \mathrm{X}$ PrimeSTAR GC was used instead of $5.0 \mu \mathrm{L} 5 \mathrm{X}$ PrimeSTAR buffer in condition 4 . All other reagents were consistent with condition 4 , except for the $\mathrm{dH}_{2} \mathrm{O}$ volume.

The PCR program consisted of an initial denaturizing step at $95^{\circ} \mathrm{C}$ for $5 \mathrm{~min}$, followed by 35 cycles of $94^{\circ} \mathrm{C}$ for $30 \mathrm{~s}, 55^{\circ} \mathrm{C}$ for $30 \mathrm{~s}$, and $72^{\circ} \mathrm{C}$ for $30 \mathrm{~s}$, and a final incubation step at $72^{\circ} \mathrm{C}$ for $5 \mathrm{~min}$. The DNA fragments were separated on $4 \%$ agarose gel and stained with ethidium bromide.

When digestion was needed, the PCR products were first purified by MiniBEST DNA fragment purification kit from TaKaRa, diluted with $20.0 \mu \mathrm{L} 1 \mathrm{X}$ digestion buffer containing $1 \mathrm{U}$ restriction enzyme, followed by incubation for $4 \mathrm{~h}$ at $37^{\circ} \mathrm{C}$ in a water or air bath. After the digestion, the samples were separated by gel electrophoresis on $4 \%$ agarose gel or $10 \%$ polyacrylamide gel (PAGE). All restriction enzymes were supplied from New England Biolabs.

\section{RESULTS}

\section{Amplification of tms 5 fragments with GC-rich PCR buffers}

Obtaining bright and specific PCR products are a prerequisite for the development of dCAPS. To amplify the mutation site of tms 5 , the 14 primer sets listed in Table 2 were tested under regular PCR conditions (condition 1, as described above). Five of 14 primer sets resulted in weak PCR bands (Figure 1A). Both primer sets 1 and 2 had a single band that was difficult to see after digestion. Primer sets 9 and 10 had bands with molecular weights inconsistent with the expected results. Primer set 11 had one clear band, but the fragment could not be digested using ScaI. Furthermore, the sequencing results for DNA extracted using primer set 11 showed that it had no homology with $t m s 5$, indicating that the S6/T3 primer set (set number 11) exhibited off-target effects. These results indicate that regular reaction conditions are not able to yield desirable results for $t m s 5$.

Then, we detected several CpG islands in TMS5. The expected PCR amplification region for tms 5 has a $\mathrm{CpG}$ island with a GC-ratio reaching $74 \%$, which may result in difficult conditions for conventional PCR procedures. To optimize the PCR conditions at these GCrich regions, we tried four different kinds of PCR buffers and two kinds of DNA polymerase to form four alternative reaction conditions (conditions 2-5), as described above. Figure 1B-E illustrates the electrophoresis results of the amplified PCR products from conditions 2-5.

Figure 1B showed that seven lanes contained relatively bright bands under condition 2 (rTaq in 2X GC buffer I). Five primer sets $(1,2,3,4$, and 11) showed a single band of the expected size. For primer sets 7 and 8 , apart from a target band, there were also some unexpected bands. The other primer combinations showed no bands. To confirm the amplified PCR results, each of the target bands was collected for sequence analysis. The results showed that the fragments taken from primer sets 1,2,3, and 4 were consistent with that of tms 5 gene, whereas primer set 11 still showed an off-target phenomenon.

Under the condition 3 reaction (rTaq in 2X GC buffer II), not only primer sets 1, 2, 3, 4 and 11 amplified a single band, but also primer sets 7 and 8 showed the same results (Figure 1C). The subsequent sequence analysis confirmed that all lanes were correct, except lane 11.

The results under conditions 4 and 5 (PrimeSTAR HS DNA polymerase in 5X

Genetics and Molecular Research 15 (3): gmr.15038512 

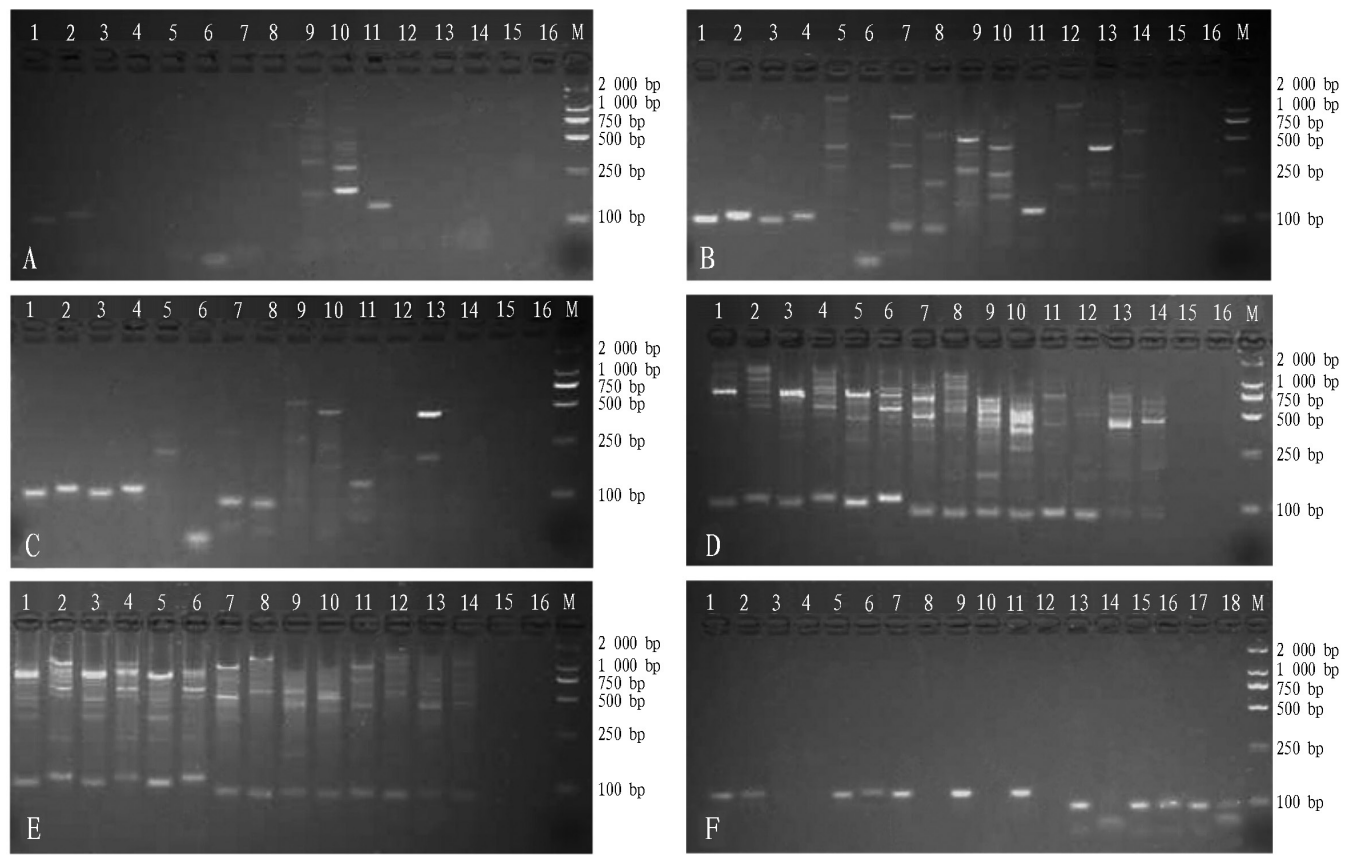

Figure 1. Results of PCR amplifications under different reaction conditions and digestions. A.-E. PCR results from conditions 1-5 are presented (as described in the material and methods-section). The number of each lane corresponds with the primer set numbers in Table 2. F. Digestion results of PCR amplicons using different restriction enzymes. Lanes 1-6 are primer set number 2, lanes 7-12 are primer set number 4, and lanes 13-18 are primer set number 7 . In each set, the DNA templates were derived from different rice varieties. The template in lanes $1,2,7,8,13$, and 14 came from 1892s; the template in lanes 3, 4, 9, 10, 15, and 16 came from Yangdao 6; and the template in lanes 5, 6, 11, 12,17, and 18 came from the $\mathrm{F}_{1}$ hybrid of $1892 \mathrm{~s}$ and Yangdao 6 . In $\mathbf{F}$, lanes with odd numbers $(1,3,5,7,9,11,13,15$, and 17$)$ are undigested PCR products, whereas lanes with even numbers $(2,4,6,8$, $10,12,14,16$, and 18) are digested PCR products. Lane $M$ indicates molecular weight ladder of DL2000 (TaKaRa).

PrimeSTAR buffer and 2X PrimeSTAR GC buffer, respectively) are presented in Figures 1DE. Our results suggest that all lanes could amplify the target 100-bp band, indicating that the PrimeSTAR HS DNA polymerase has a strong ability for tms 5 PCR amplification. However, some unexpected bands with too high molecular weights also appeared in the gel. This suggests that the reaction conditions for this DNA polymerase need to be further adjusted.

The PCR amplification results of the mismatched primers could be found in Figure 1A-E. For selective primers with two mismatched bases (S3, S5, S6, and S7), no ideal band was obtained under any of the five amplification conditions. For primers with one mismatched base, three primers (S1, S2, and S4) could amplify a single target band either under condition 2 or 3 (Figure 1B and C). These results suggest that mismatches close to the 3 ' end of the primers have a relatively great impact on the PCR amplification results.

\section{Digestion of amplified products of $t m s 5$ with $R s a I$}

Since the selective primers with a single mismatch (S1, S2, and S4) could amplify ideal bands with all target primers with rTaq in GC buffer II, we selected two (T2 and T3) of 
the four target primers to give three primer sets (sets 2,4 , and 7) to amplify the $F_{1}$ hybrid of Huiliangyou 6 and its parents 1892s and Yangdao 6. The amplified products were digested with the corresponding restriction enzymes as listed in Table 2.

When primer set 2 was used to amplify the genomic DNA of 1892s, Yangdao 6, and their $\mathrm{F}_{1}$ hybrid, the PCR products from 1892s and the $\mathrm{F}_{1}$ hybrid could not be digested with MaeI, whereas Yangdao 6 gave no PCR product (Figure 1F, lanes 1-6). For the PCR products amplified by primer set 4, target bands were bright but disappeared after MboII digestion (Figure 1F, lanes 8, 10, and 12), suggesting that MboII may have some effect of asterisk and was not suitable for the development of dCAPS. For PCR products amplified using primer set 7, three bright bands from each template were obtained with the expected molecular weights. After RsaI digestion, the molecular weight of $1892 \mathrm{~s}$ amplicon was reduced significantly (Figure $1 \mathrm{~F}$, lane 14), compared with the band without digestion (Figure $1 \mathrm{~F}$, lane 13). Amplicons of Yangdao 6 showed no change and the $F_{1}$ hybrid had two bands (Figure $1 \mathrm{~F}$, lane 18), which are the features of a co-dominant marker. This encouraging PCR amplification result from primer set 7 is consistent with the design purpose. The bands digested with RsaI did not have a sufficiently high resolution on the agarose gel, due to their small molecular size. Therefore, a 10\% PAGE was used to separate the low molecular weight DNA fragments (Figure 2). Among these, lane 4 is the completely digested tms 5 in 1892s, lane 5 is undigested in Yangdao 6, whereas lane 6 is heterozygous $\mathrm{F}_{1}$ hybrid (tms5/TMS5) whose complementary bands came from its parents.

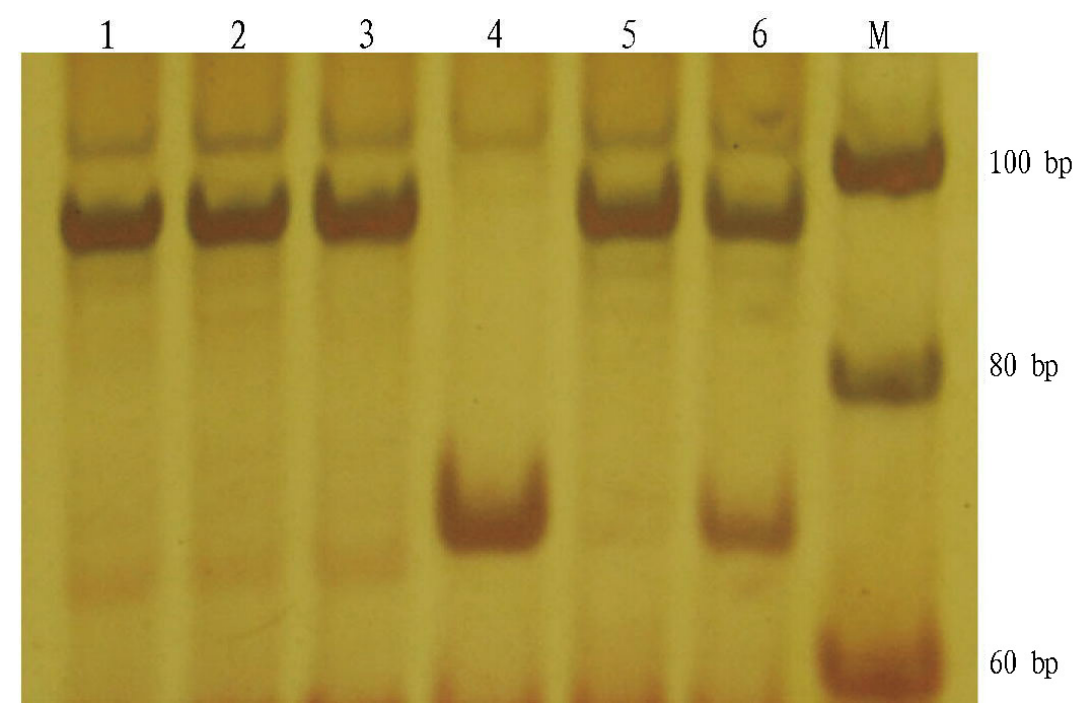

Figure 2. RsaI digested PCR products using primer set 7 on 10\% PAGE. Lanes 1-3 are undigested PCR products and lanes 4- 6 are digested with RsaI (lanes 1 and $4=1892 \mathrm{~s}$, lanes 2 and $5=$ Yangdao 6 , lanes 3 and $6=\mathrm{F}_{1}$ hybrid of 1892s and Yangdao 6, lane $M=$ molecular weight marker). The molecular weights of the DNA fragments are 94 (TMS5, uncleaved type) and $68 \mathrm{bp}$ (tms5, cleaved type), respectively.

The digested PCR products amplified with primer set 7 were unclear in agarose gel, due to their low molecular weight. Since agarose gel electrophoresis is popular in many labs, we improved the applicability by designing more target primers (T5, T6, and T7, see Table 1) to increase the molecular weights of the PCR products. These newly designed target primers

Genetics and Molecular Research 15 (3): gmr.15038512 
were well matched with selective primer S4 to amplify genomic DNAs of 1892s, Yangdao 6, and $\mathrm{F}_{1}$ hybrid in buffer condition 3 (Figure 3). Only primer set S4/T7 resulted in a clear 172-bp target band, whereas the S4/T5 and S4/T6 primer sets resulted in multiple unexpected bands. When the 172-bp PCR products of S4/T7 were digested using RsaI, our results indicated that the 1892s amplified PCR product could be digested, but not that of Yangdao 6. The digested $F_{1}$ hybrid PCR product resulted in heterozygous double bands (Figure 4). All digested bands were clear and polymorphic on the agarose gel. The sequencing results also suggested that the $172 \mathrm{bp} \mathrm{S4/T7} \mathrm{fragment} \mathrm{was} \mathrm{consistent} \mathrm{with} \mathrm{tms5.} \mathrm{We} \mathrm{named} \mathrm{the} \mathrm{new} \mathrm{dCAPS} \mathrm{marker}$ dCAPS-172.

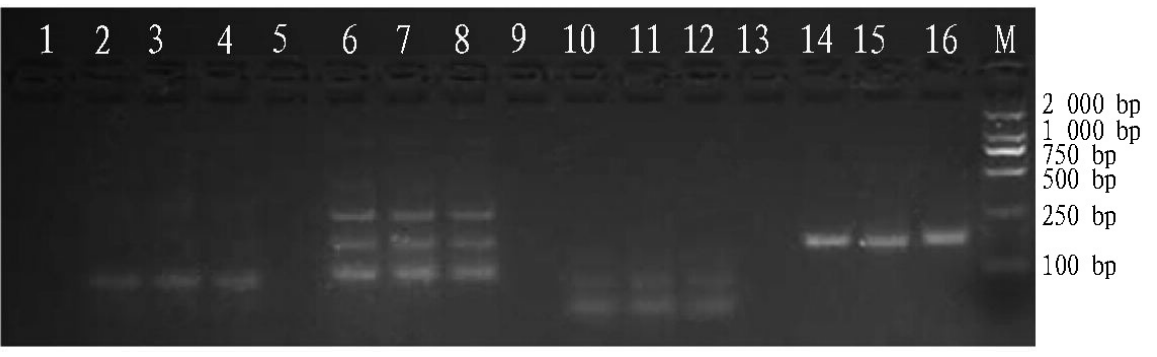

Figure 3. PCR amplification of F4 matching with newly designed target primers. Lanes 1, 5, 9, and 13 are negative controls; lanes 2-4, 6-7, 10-12, and 14-16 are PCR products amplified with primer sets S4/T3, S4/T5, S4/T6, and $\mathrm{S} 4 / \mathrm{T} 7$, respectively. Lanes 2, 6, 10, and $14=1892 \mathrm{~s}$; lanes 3, 7, 11, and $15=$ Yangdao 6; lanes 4, 8, 12, and $16=\mathrm{F}_{1}$ hybrid of 1892 s and Yangdao 6 ; lane $M=$ molecular weight ladder.

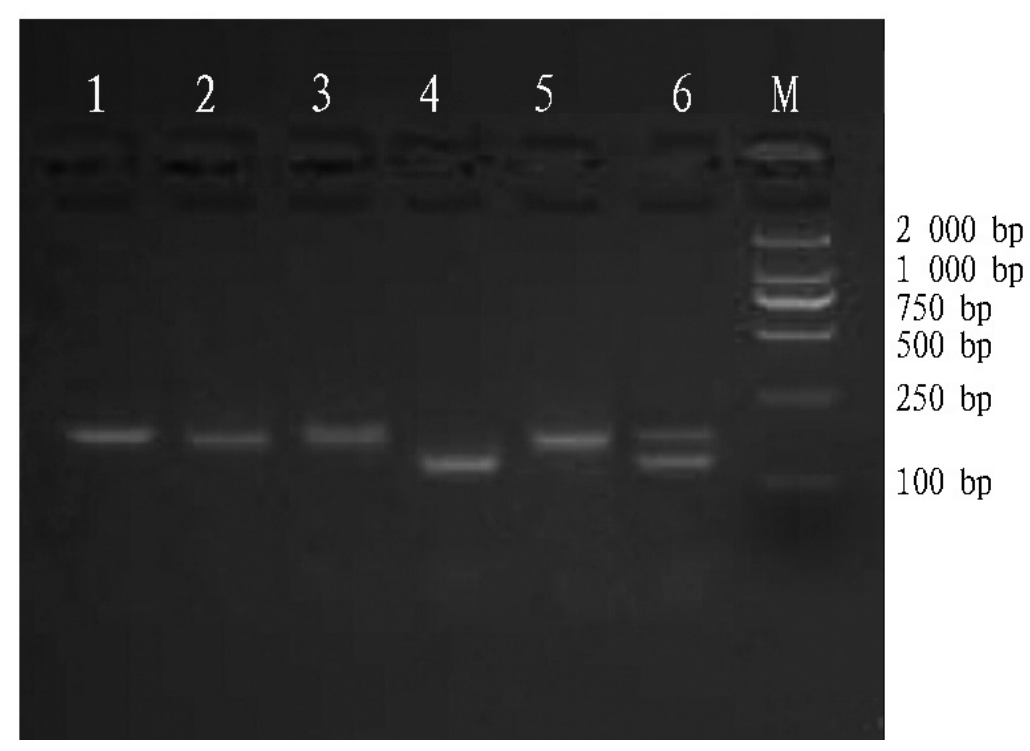

Figure 4. Digested PCR products of S4/T8 separated by 4\% agarose gel electrophoresis. Lanes 1-3 are undigested amplicons from 1892s, Yangdao 6, and $\mathrm{F}_{1}$ hybrid of 1892s and Yangdao 6. Lanes 4-6 are RsaI digested amplicons from 1892s, Yangdao 6, and $\mathrm{F}_{1}$ hybrid. Lane $M=$ molecular weight ladder. The molecular weight for the restricted DNA fragments are 172 (TMS5, uncleaved type) and $146 \mathrm{bp}$ (tms 5, cleaved type). 
PCR product purifications of restriction digestions are usually arduous, which can limit their usage in high-throughput detection. To simplify the process, $2 \mathrm{U} R s a \mathrm{I}$ were directly added to the reaction tubes after the PCR amplification without digestion buffers. The reactions were incubated at $37^{\circ} \mathrm{C}$, varying the incubation time from 1 to $16 \mathrm{~h}$. Amplicons could be cleaved correctly in PCR 2X GC buffer II system with no electrophoresis profile changes among the extended digestion times, which indicates that $R s a \mathrm{I}$ is suitable for large-scale screening.

\section{Detecting tms 5 with the dCAPS-172 marker}

The $\mathrm{F}_{2}$ population derived from the cross between 1892s and Yangdao 6 was selected to analyze the reliability of the new dCAPS- 172 marker. We investigated $192 \mathrm{~F}_{2}$ population plants and found that 43 plants were sterile, showing the same bands as 1892s (cleaved type). The rest of the plants (149 plants) were fertile. Among these, 52 resulted in the same bands as Yangdao 6 when undigested by RsaI. The other 97 fertile plants showed heterozygous bands, like their parents. The results of the molecular detection were completely consistent with the phenotypic identification.

We also tested the dCAPS-172 marker on the other TGMS lines. Among these, amplicons from 14 tms5-type TGMS lines sequenced for the $R N Z$ gene could be cleaved correctly by RsaI, but none of the USDA Rice Mini-Core Collections could be digested by $R s a \mathrm{I}$. We also retrieved haplotypes from the $R s a \mathrm{I}$ restriction site (GTAG, Chr2:63974106397413, MSU 6.0) from sequencing data of 1479 rice accessions including landraces and improved varieties from 73 countries (http://ricevarmap.ncpgr.cn/). Only two haplotypes (GGCG and GTCG) were found that could not be digested by RsaI. In conclusion, these results suggest that the dCAPS- 172 marker can be used to test tms 5 allele accurately and that it is suitable for marker-assisted selection.

\section{Purity detection of hybrid rice}

Ensuring the purity of hybrid rice is important for hybrid rice production. During seed production of two-line hybrid rice, temperature fluctuations results in a potential problem when the female parent produces selfed seeds in addition to hybrid seeds. In theory, tms 5 type hybrid rice is heterozygous on the locus, but inbred rice is homozygous. We believe that co-dominant dCAPS markers could be used to detect the purity of hybrid rice. To verify this hypothesis, 200 plants of two cultivars, Wandao 153 and Huiliangyou 6, were planted in a local paddy field. DNA samples from each cultivar were extracted and detected using the dCAPS-172 marker. In four plants of Wandao 153 and seven plants of Huiliangyou 6, a single $146 \mathrm{bp}$ fragment was observed. This was the same band as that observed in cultivar 1892s. Pollen from these 11 plants could not be stained using the $\mathrm{I}_{2}-\mathrm{KI}$ solution. In other plants that resulted in heterozygous double bands, the pollen was stained dark by the $\mathrm{I}_{2}$-KI solution. These results indicated that the dCAPS-172 marker could be a useful tool for identifying the purity of tms5-type hybrid rice.

\section{DISCUSSION}

In China, both the pms 3 and the tms 5 genes are used as sterile male parents in two-line hybrid rice breeding. In practice, tms 5 appears more popular than $p m s 3$ in rice seed production.

Genetics and Molecular Research 15 (3): gmr.15038512 
Zhou et al. (2014) found that tms 5-containing TGMS lines accounted for at least $71 \%$ of all twoline hybrid rice cultivars and $83.8 \%$ of all the land used to grow two-line hybrid rice in China. Zhang et al. (2015) revealed that most of the two-line sterile lines in China harbored tms5. Accordingly, tms 5 serves as the major TGMS genetic resource for two-line hybrid rice breeding.

Genetic markers are DNA fragments that are associated with a certain location within the genome. The use of functional genetic markers is a precise way for genetic identification, because it detects genetic disorders that lead to phenotypic changes. Both CAPS and dCAPS markers are inexpensive and present an effective way to make point mutations or to transform SNPs to PCR-based markers. To make dCAPS markers, the design of selective primers is the first step. In this study, we designed seven selective primers, including three primers with one mismatched base and four primers with two mismatched bases. All primers with a single mismatch could successfully amplify the target band under specific conditions. However, of the four primers with two mismatched bases, three resulted in no amplified product, and one exhibited off-target results. Among the three primers with a single mismatch, two had a mismatched base located at the last position, by the 3' end, and the other had a mismatched base located at the second last position of the 3' end of the primer. The mismatches included $\mathrm{G} \rightarrow \mathrm{C}, \mathrm{T} \rightarrow \mathrm{A}$, and $\mathrm{C} \rightarrow \mathrm{G}$. Regardless of the PCR settings, the PCR amplification results from this study suggest that the number of mismatched bases has a greater impact than the mismatch position or the base type.

For some genomic loci that are difficult to amplify, the expected results could be obtained by optimizing the PCR reagents. In this study, 2X GC buffer II was the most suitable buffer for amplification of tms5. PrimeSTAR HS DNA polymerase was found to be better at overcoming the mismatches than $r$ Taq DNA polymerase. The seven selective primers with one or two mismatch bases were able to amplify target bands by PrimeSTAR HS DNA polymerase, although they often resulted in off-target PCR products. If the nonspecific amplification did not affect the detection results, PrimeSTAR HS polymerase was more suitable for dCAPS than $r$ Taq DNA polymerase, although it is a bit more expensive.

Not all the restriction endonucleases investigated here were found to be suitable to digest dCAPS marker amplicons. In this study, we designed dCAPS markers with three different restriction enzymes that digest only the tms 5 mutation but not wild type amplicons, in order to avoid the possibilities of false positives. MaeI led to incomplete digestion, whereas MboII resulted in nonspecific digestion. Only RsaI both cut well and resulted in the expected output.

In summary, we developed a new dCAPS marker that is able to rapidly and accurately distinguish between tms 5 and TMS5. We have shown that the dCAPS-172 marker can be applied in marker-assisted breeding selection as well as be used to identify the purity of tms 5type hybrid rice seeds. The development of this marker can also provide a useful reference for the design of other dCAPS markers.

\section{Conflicts of interest}

The authors declare no conflict of interest.

\section{ACKNOWLEDGMENTS}

Research supported by the National Natural Science Foundation of China (\#31201276), the Natural Science Foundation of Anhui Province (\#1408085MC55), the Science and

Genetics and Molecular Research 15 (3): gmr.15038512 
Technology Research of Anhui Province (\#1401032001), the Science and Technology Key Projects of Anhui Province (\#1604a0702008) and the Science and Technology Innovation Team of Anhui Agricultural Academy of Sciences (\#14C0101).

\section{REFERENCES}

Agrama HA, Yan WG, Lee F, Fjellstrom R, et al. (2009). Genetic assessment of a mini-core subset developed from the USDA rice Genebank. Crop Sci. 49: 1336-1346. http://dx.doi.org/10.2135/cropsci2008.06.0551

Cao XF, Yang YZ, Zhou M and Hu XC (2011). A rapid method to detect rice thermo-sensitive sterile gene tms5. China, patent application number: 201110292922.0, 2013-04-10.

Deng H, Shu F and Yuan D (1999). An overview of research and utilization of Annong S-1. Hybrid Rice 14: 1-3.

Ding J, Lu Q, Ouyang Y, Mao H, et al. (2012). A long noncoding RNA regulates photoperiod-sensitive male sterility, an essential component of hybrid rice. Proc. Natl. Acad. Sci. USA 109: 2654-2659. http://dx.doi.org/10.1073/ pnas. 1121374109

Dong NV, Subudhi PK, Luong PN, Quang VD, et al. (2000). Molecular mapping of a rice gene conditioning thermosensitive genic male sterility using AFLP, RFLP, and SSR techniques. Theor. Appl. Genet. 100: 727-734. http://dx.doi. org/10.1007/s001220051345

Fan Y and Zhang Q (2014). Understanding a key gene for thermosensitive genic male sterility in rice. Sci. China Life Sci. 57: 1241-1242. http://dx.doi.org/10.1007/s11427-014-4763-6

Li H, Li J, Cong XH, Duan YB, et al. (2013). A high-throughput, high-quality plant genomic DNA extraction protocol. Genet. Mol. Res. 12: 4526-4539. http://dx.doi.org/10.4238/2013.October.15.1

Li X, Yan W, Agrama H, Hu B, et al. (2010). Genotypic and phenotypic characterization of genetic differentiation and diversity in the USDA rice mini-core collection. Genetica 138: 1221-1230. http://dx.doi.org/10.1007/s10709-0109521-5

Nas TMS, Sanchez DL, Diaz GQ, Mendioro MS, et al. (2005). Pyramiding of thermosensitive genetic male sterility (TGMS) genes and identification of a candidate tms 5 gene in rice. Euphytica 145: 67-75. http://dx.doi.org/10.1007/ s10681-005-0206-6

Neff MM, Turk E and Kalishman M (2002). Web-based primer design for single nucleotide polymorphism analysis. Trends Genet. 18: 613-615. http://dx.doi.org/10.1016/S0168-9525(02)02820-2

Peng HF, Chen XH, Lu YP, Peng YF, et al. (2010). Fine mapping of a gene for non-pollen type thermosensitive genic male sterility in rice (Oryza sativa L.). Theor. Appl. Genet. 120: 1013-1020. http://dx.doi.org/10.1007/s00122-009-1229-5

Qi Y, Liu Q, Zhang L, Mao B, et al. (2014). Fine mapping and candidate gene analysis of the novel thermo-sensitive genic male sterility tms $9-1$ gene in rice. Theor. Appl. Genet. 127: 1173-1182. http://dx.doi.org/10.1007/s00122-014-2289-8

Shi MS (1985). The discovery and study of the photosensitive recessive male-sterile rice. Sci. Agric. Sin. 2: 44-48.

Xu J, Wang B, Wu Y, Du P, et al. (2011). Fine mapping and candidate gene analysis of ptgms2-1, the photoperiodthermo-sensitive genic male sterile gene in rice (Oryza sativa L.). Theor. Appl. Genet. 122: 365-372. http://dx.doi. org/10.1007/s00122-010-1452-0

Yang JB, Lu XZ, Ni JL, Ma H, et al. (2011). Development and application of a closely linked marker with rice thermosensitive sterile gene tms5. China, patent number: $\mathrm{zl}$ 2001110326770.1, 2012-06-20.

Zhang HL, Huang JZ, Liu QL, Nawaz Z, et al. (2014a). Characterization of an RNase Z nonsense mutation identified exclusively in environment-conditioned genic male sterile rice. Mol. Breed. 34: 481-489. http://dx.doi.org/10.1007/ $\underline{\text { s11032-014-0051-1 }}$

Zhang HL, Huang JZ, Chen XY, Tan YY, et al. (2014b). Competitive amplification of differentially melting amplicons facilitates efficient genotyping of photoperiod- and temperature-sensitive genic male sterility in rice. Mol. Breed. 34: 1765-1776. http://dx.doi.org/10.1007/s11032-014-0136-x

Zhang HL, Chen XY, Huang JZ, Zhi-guo E, et al. (2015). Identification and transition analysis of photo-/thermosensitive genic male sterile genes in two-line hybrid rice in China. Sci. Agric. Sin. 48: 1-9. http://dx.doi.org/10.1017/ $\underline{\mathrm{S} 0021859615001008}$

Zhou H, Liu Q, Li J, Jiang D, et al. (2012). Photoperiod- and thermo-sensitive genic male sterility in rice are caused by a point mutation in a novel noncoding RNA that produces a small RNA. Cell Res. 22: 649-660. http://dx.doi. org/10.1038/cr.2012.28

Zhou H, Zhou M, Yang Y, Li J, et al. (2014). RNase Z(S1) processes UbL40 mRNAs and controls thermosensitive genic male sterility in rice. Nat. Commun. 5: 4884-4892. http://dx.doi.org/10.1038/ncomms5884

Genetics and Molecular Research 15 (3): gmr.15038512 